\title{
Generalized proportional fractional integral Hermite-Hadamard's inequalities
}

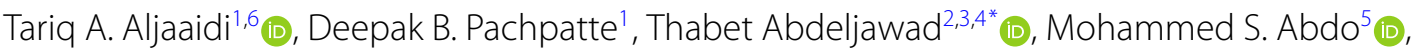 \\ Mohammed A. Almalahi ${ }^{6}$ and Saleh S. Redhwan ${ }^{1}$
}

\author{
*Correspondence: \\ tabdeljawad@psu.edu.sa \\ ${ }^{2}$ Department of Mathematics and \\ Sciences, Prince Sultan University, \\ Riyadh, Saudi Arabia \\ ${ }^{3}$ Department of Medical Research, \\ China Medical University, Taichung, \\ 40402, Taiwan \\ Full list of author information is \\ available at the end of the article
}

\begin{abstract}
The theory of fractional integral inequalities plays an intrinsic role in approximation theory also it has been a key in establishing the uniqueness of solutions for some fractional differential equations. Fractional calculus has been found to be the best for modeling physical and engineering processes. More precisely, the proportional fractional operators are one of the recent important notions of fractional calculus. Our aim in this research paper is developing some novel ways of fractional integral Hermite-Hadamard inequalities in the frame of a proportional fractional integral with respect to another strictly increasing continuous function. The considered fractional integral is applied to establish some new fractional integral Hermite-Hadamard-type inequalities. Moreover, we present some special cases throughout discussing this work.
\end{abstract}

MSC: 26D10;26D15; 34K38; 26A33

Keywords: Hermite-Hadamard inequalities; Fractional inequalities; $\psi$-proportional fractional operators

\section{Introduction}

The theory of fractional calculus, which is interested in differential and integral operators of noninteger orders, is just about as old as the classical calculus, which deals with integer orders. Since not the totality of the real phenomena can be modeled by employing the operators in the classical calculus, scientists and authors looked for generalizations of these operators. As of now, we can say that the theory of fractional calculus is of considerable interest for a large number of researchers. There is a large amount of exploration and literature especially on the classical fractional calculus like the definitions of RiemannLiouville (RL) and Caputo. The RL derivative is a general common concept and, in some definitions, the most uniform and natural. However, it has some disadvantages when employing in modeling physical problems in view of the fact that the requisite initial conditions are themselves fractional, which is perhaps inappropriate for physical conditions. The Caputo derivative has the advantage of being suitable for physical conditions because it requires only initial conditions of the traditional type [1].

(c) The Author(s) 2021. This article is licensed under a Creative Commons Attribution 4.0 International License, which permits use, sharing, adaptation, distribution and reproduction in any medium or format, as long as you give appropriate credit to the original author(s) and the source, provide a link to the Creative Commons licence, and indicate if changes were made. The images or other third party material in this article are included in the article's Creative Commons licence, unless indicated otherwise in a credit line to the material. If material is not included in the article's Creative Commons licence and your intended use is not permitted by statutory regulation or exceeds the permitted use, you will need to obtain permission directly from the copyright holder. To view a copy of this licence, visit http://creativecommons.org/licenses/by/4.0/. 
Be that as it may, these are by no means the only methods of describing and defining fractional calculus. In fact, there are multiple definitions of fractional operators; RL, Caputo, Hilfer, Riesz, Hadamard, Erdélyi-Kober, Katugampola, Caputo-Fabrizio, Atangana-Baleanu definitions are just a few examples to make reference to [2-6]. Each concept has its own conditions and rules, due to which a some of these definitions are not identical to each other. In practice, the physical framework under regard decides the placement of an appropriate fractional operator. As a consequence, researchers have introduced various definitions of inequivalent fractional operators, every one of which is helpful in its own specific context. In view of the foregoing, it is natural for mathematicians to develop and explore fractional operators that are generalized categories of the current specific cases. For some applications and recent contributions of fractional calculus, we refer the readers to [7-11].

Our focus here is on mathematical inequalities, which are critically important in many useful applications, especially, in classical differential equations and integrals. Accordingly, in the last two centuries, several useful mathematical inequalities have been presented by mathematicians. Among mathematical inequalities, one inequality has a wellknown area along with the inequality theory. It is the well-known Hermite-Hadamard inequality. The first suggest of this inequality is attributed to Hermite (1881), but until 1893, this result was not taken into a consideration in the literature and was not vastly known as Hermite's inequality. Beckenbach [12] wrote that the inequality

$$
\varphi\left(\frac{a+b}{2}\right) \leq \frac{1}{(a-b)} \int_{a}^{b} \varphi(u) d u \leq \frac{\varphi(a)+\varphi(b)}{2}, \quad a, b \in \mathbb{R}, a<b
$$

which holds for convex function $\varphi$, was proved by Hadamard in (1893) and called it the Hermite-Hadamard inequality, and since then, it had several generalizations and extensions for univariable, bivariable, and multivariable convex functions (see [13]). Significantly, this inequality has become the focus of attention of many researchers and mathematicians due to its important applications and wide uses in many fields. As a result, a lot of works have appeared that include generalizations and extensions of this inequality; see [14-16].

Mathematical inequalities had to take their share of the theory of fractional calculus, concerning the Hermite-Hadamard inequality. Sarikaya et al. [17] in 2013 presented an RL-type fractional integral inequality formula for convex functions.

Theorem 1.1 ([17]) Let $\varphi:[a, b] \longrightarrow \mathbb{R}$ be a positive convex function on $[a, b]$ with $0 \leq a<$ $b$. Then for all $\mu>0$, we have the following inequalities:

$$
\frac{\varphi(a)+\varphi(b)}{2} \leq \frac{\Gamma(\mu+1)}{2(a+b)^{\mu}}\left[\mathcal{I}_{a^{+}}^{\mu} \varphi(b)+\mathcal{I}_{b^{-}}^{\mu} \varphi(a)\right] \leq \varphi\left(\frac{a+b}{2}\right)
$$

Chen [18] in 2016 proved the following Riemann-Liouville fractional integral HermiteHadamard type inequalities.

Theorem 1.2 Let $\varphi:[a, b] \longrightarrow \mathbb{R}$ with $0 \leq a<b$ be a positive twice differentiable function on $(a, b)$, which is integrable $[a, b]$. If $\varphi^{\prime \prime}$ is bounded in $[a, b]$, then we have the following 
inequalities:

$$
\begin{aligned}
& \frac{\mu C_{1}}{2(b-a)^{\mu}} \int_{a}^{\left(\frac{a+b}{2}\right)}\left(\frac{a+b}{2}-u\right)^{2}\left\{(b-u)^{\mu-1}+(u-a)^{\mu-1}\right\} d u \\
& \quad \leq \frac{\Gamma(\mu+1)}{2(b-a)^{\mu}}\left\{\mathcal{I}_{a^{+}}^{\mu} \varphi(b)+\mathcal{I}_{b^{-}}^{\mu} \varphi(a)\right\}-\varphi\left\{\frac{a+b}{2}\right\} \\
& \quad \leq \frac{\mu C_{2}}{2(b-a)^{\mu}} \int_{a}^{\left(\frac{a+b}{2}\right)}\left(\frac{a+b}{2}-u\right)^{2}\left\{(b-u)^{\mu-1}+(u-a)^{\mu-1}\right\} d u .
\end{aligned}
$$

Theorem 1.3 Let $\varphi:[a, b] \longrightarrow \mathbb{R}$ with $0 \leq a<b$ be a positive twice differentiable function on $(a, b)$, which is integrable function on $[a, b]$. If $\varphi^{\prime \prime}$ is bounded in $[a, b]$, then we have the following inequalities:

$$
\begin{aligned}
& \frac{-\mu C_{2}}{2(b-a)^{\mu}} \int_{a}^{\left(\frac{a+b}{2}\right)}[(b-u)(u-a)]\left\{(b-u)^{\mu-1}+(u-a)^{\mu-1}\right\} d u \\
& \quad \leq \frac{\Gamma(\mu+1)}{2(b-a)^{\mu}}\left\{\mathcal{I}_{a^{+}}^{\mu} \varphi(b)+\mathcal{I}_{b^{-}}^{\mu} \varphi(a)\right\}-\frac{\varphi(a)+\varphi(b)}{2} \\
& \quad \leq \frac{-\mu C_{1}}{2(b-a)^{\mu}} \int_{a}^{\left(\frac{a+b}{2}\right)}[(b-u)(u-a)]\left\{(b-u)^{\mu-1}+(u-a)^{\mu-1}\right\} d u .
\end{aligned}
$$

Chen [18] also proved the fractional integral Hermite-Hadamard inequality (1.2) without using the convexity of the function $\varphi$.

Liu [19] in (2019) presented inequality (1.2) with respect to another positive increasing monotone function.

Theorem 1.4 Let $\varphi:[a, b] \longrightarrow \mathbb{R}$ with $0 \leq a<b$ be a positive convex function on $[a, b]$, and let $\psi(u)$ be an increasing positive function having a continuous derivative on $(a, b)$. Then we have the following inequalities:

$$
\begin{aligned}
\varphi\left(\frac{a+b}{2}\right) & \leq \frac{\Gamma(\mu+1)}{2(b-a)^{\mu}}\left\{{ }^{\psi} \mathcal{I}_{\left\{\psi^{-1}(a)\right\}^{+}}^{\mu}(\varphi \circ \psi)\left(\psi^{-1}(b)\right)+{ }^{\psi} \mathcal{I}_{\left\{\psi^{-1}(b)\right\}^{-}}^{\mu}(\varphi \circ \psi)\left(\psi^{-1}(a)\right)\right\} \\
& \leq \frac{\varphi(a)+\varphi(b)}{2} .
\end{aligned}
$$

As an application of the Hermite-Hadamard inequality, Nowicka and Witkowski [20] showed how certain its improvements can be applied to some (not necessarily convex) planar figures and three-dimensional bodies satisfying some kind of regularity. Dahmani [21] in (2010) employed concave functions to present Hermite-Hadamard-type inequalities using the Riemann-Liouville fractional integral. Set et al. in (2014) studied the HermiteHadamard inequality for the second sense of s-convex functions, which was proved by Dragomir et al. [22], and m-convex functions employing the fractional integrals. Noor et al. [23] in (2015) by using the q-differentiable convex functions and q-differentiable quasi-convex functions derived some quantum estimates for Hermite-Hadamard-type inequalities. Agarwal et al. [24] in (2017), employing the (k,s)-Riemann-Liouville fractional integrals, obtained some Hermite-Hadamard-type inequalities for convex functions. Khan [25] in (2018) introduced some new Hermite-Hadamard-type inequalities 
employing the convex functions, s-convex, and coordinate convex functions via the conformable fractional integral. Pshtiwan and Brevik [26] in (2020) introduced a new version of the Hermite-Hadamard inequality for the Riemann-Liouville fractional integrals. For $n$-polynomial harmonically convex functions, Awan et al. [27] in the same year proved some new Hermite-Hadamard-type inequalities. Also, Chudziak and Ołdak [28]presented a notion of coordinated $(F, G)$-convex functions defined on an interval in $\mathbb{R}^{2}$. Recently, in (2021), Khan [29] studied the Hermite-Hadamard inequality for coordinates of convex fuzzy interval-valued functions. A lot of the authors studied the HermiteHadamard inequalities for several different types of convexity of the functions via several different types of fractional integral and derivative operators; for more detail, we refer the readers to [30-34].

Motivated by the above argumentation and inspired by the appreciated works introduced by Sarikaya [17] and Chen [18], and by taking advantage of the generalized proportional fractional integral operator concerning another strictly increasing continuous function, in this work, we establish some new generalized fractional integral HermiteHadamard inequalities in the frame of generalized proportional fractional integral operators, which, to the best of our knowledge, have not yet been considered until the current day. With the help of the considered proportional fractional operator, we also introduce some fractional integral Hermite-Hadamard-type inequalities. Moreover, we enrich this work by discussing some particular cases related to the current research paper.

The organization of this research paper is as follows. Throughout the next section, we point some definitions, notations, and introductory acquaintance used in this work. Sections 3 and 4 are devoted to our major results on Hermite-Hadamard inequalities and Hermite-Hadamard-type inequalities, respectively.

\section{Essential preliminaries}

Here we recall some basic definitions and properties of elementary fractional integral operators.

Definition 2.1 A function $\varphi:([a, b] \subseteq \mathbb{R}) \rightarrow \mathbb{R}$ is said to be a convex function if

$$
\varphi(\lambda r+(1-\lambda) s) \leq \lambda \varphi(r)+(1-\lambda) \varphi(s)
$$

for all $r, s \in[a, b]$ and $\lambda \in[0,1]$. We say that $\varphi$ is concave if inequality (2.1) is reversed.

Definition 2.2 ([2]) For an integrable function $\varphi$ on $[a, b]$ and $a \geq 0$, we have, for all $\mu>0$,

$$
\mathcal{I}_{a^{+}}^{\mu} \varphi(t)=\frac{1}{\Gamma(\mu)} \int_{a}^{t}(t-u)^{\mu-1} \varphi(u) d u, \quad u>a
$$

and

$$
\mathcal{I}_{b^{-}}^{\mu} \varphi(t)=\frac{1}{\Gamma(\mu)} \int_{t}^{b}(u-t)^{\mu-1} \varphi(u) d u, \quad t<b,
$$

where $\Gamma(\mu)=\int_{0}^{\infty} e^{-x} x^{\mu-1} d x$ is the gamma function, and $\mathcal{I}_{a^{+}}^{0} \varphi(t)=\mathcal{I}_{b^{-}}^{0} \varphi(t)=\varphi(t)$. The functions $\mathcal{I}_{a^{+}}^{\mu} \varphi(t)$ and $\mathcal{I}_{b^{-}}^{\mu} \varphi(t)$ are called the left- and right-sided Riemann-Liouville fractional integrals, respectively, of the function $\varphi$ for the order $\mu$. 
Definition $2.3([2,3])$ For an integrable function $\varphi$ on the interval $\Lambda$ and for an increasing function $\psi \in C^{1}(\Lambda, \mathbb{R})$ such that $\psi^{\prime}(t) \neq 0, t \in \Lambda$, we have, for all $\mu>0$,

$$
{ }^{\psi} \mathcal{I}_{a^{+}}^{\mu} \varphi(t)=\frac{1}{\Gamma(\mu)} \int_{a}^{t} \psi^{\prime}(u)[\psi(t)-\psi(u)]^{\mu-1} \varphi(u) d u
$$

and

$$
{ }^{\psi} \mathcal{I}_{b^{-}}^{\mu} \varphi(t)=\frac{1}{\Gamma(\mu)} \int_{t}^{b} \psi^{\prime}(u)[\psi(u)-\psi(t)]^{\mu-1} \varphi(u) d u .
$$

The functions ${ }^{\psi} \mathcal{I}_{a^{+}}^{\mu} \varphi(t)$ and ${ }^{\psi} \mathcal{I}_{b^{-}}^{\mu} \varphi(t)$ are called the left- and right-sided $\psi$-RiemannLiouville fractional integrals, respectively, of the function $\varphi$ for the order $\mu$.

Definition 2.4 ([35]) For an integrable function $\varphi$ and $\omega>0$, we have, for all $\mu \in \mathbb{C}$, $\operatorname{Re}(\mu) \geq 0$,

$$
\begin{aligned}
\left(D_{a^{+}}^{\mu, \omega} \varphi\right)(t) & =D^{m, \omega} \mathcal{I}_{a^{+}}^{m-\mu, \omega} \varphi(t) \\
& =\frac{D_{t}^{m, \omega}}{\omega^{m-\mu} \Gamma(m-\mu)} \int_{a}^{t} \exp \left[\frac{\omega-1}{\omega}(t-u)\right](t-u)^{m-\mu-1} \varphi(u) d u
\end{aligned}
$$

and

$$
\begin{aligned}
\left(D_{b^{-}}^{\mu, \omega} \varphi\right)(t) & ={ }_{\gamma} D^{m, \omega} \mathcal{I}_{b^{-}}^{m-\mu, \omega} \varphi(t) \\
& =\frac{{ } D_{t}^{m, \omega}}{\omega^{m-\mu} \Gamma(m-\mu)} \int_{t}^{b} \exp \left[\frac{\omega-1}{\omega}(u-t)\right](u-t)^{m-\mu-1} \varphi(u) d u,
\end{aligned}
$$

where

$$
D^{m, \omega}=\underbrace{D^{\omega} D^{\omega} \cdots D^{\omega}}_{m \text {-times }}, \quad m=[\operatorname{Re}(\mu)]+1
$$

and

$$
\left({ }_{\gamma} D^{\omega} \varphi\right)(t)=(1-\omega) \varphi(t)-\omega \varphi^{\prime}(t), \quad{ }_{\gamma} D^{m, \omega}=\underbrace{{ }_{\gamma} D_{\gamma}^{\omega} D^{\omega} \cdots{ }_{\gamma} D^{\omega}}_{m \text {-times }} .
$$

The functions $\left(D_{a^{+}}^{\mu, \omega} \varphi\right)(t)$ and $\left(D_{b^{-}}^{\mu, \omega} \varphi\right)(t)$ are called the left- and right-sided proportional fractional derivatives, respectively, of the function $\varphi$ for the order $\mu$.

Definition 2.5 ([35]) For an integrable function $\varphi$ and $\omega>0$, we have, for all $\mu \in \mathbb{C}$, $\operatorname{Re}(\mu) \geq 0$,

$$
\left(\mathcal{I}_{a^{+}}^{\mu, \omega} \varphi\right)(t)=\frac{1}{\omega^{\mu} \Gamma(\mu)} \int_{a}^{t} \exp \left[\frac{\omega-1}{\omega}(t-u)\right](t-u)^{\mu-1} \varphi(u) d u
$$

and

$$
\left(\mathcal{I}_{b^{-}}^{\mu, \omega} \varphi\right)(t)=\frac{1}{\omega^{\mu} \Gamma(\mu)} \int_{t}^{b} \exp \left[\frac{\omega-1}{\omega}(u-t)\right](u-t)^{\mu-1} \varphi(u) d u
$$


The functions $\left(\mathcal{I}_{a^{+}}^{\mu, \omega} \varphi\right)(t)$ and $\left(\mathcal{I}_{b^{-}}^{\mu, \omega} \varphi\right)(t)$ are called the left- and right-sided proportional fractional integrals, respectively, of the function $\varphi$ for the order $\mu$.

Definition 2.6 ([36]) For an integrable function $\varphi$, a strictly increasing continuous function $\psi$ on $[a, b]$, and $\omega \in(0,1]$, we have, for all $\mu \in \mathbb{C}, \operatorname{Re}(\mu) \geq 0$,

$$
\begin{aligned}
\left({ }^{\psi} D_{a^{+}}^{\mu, \omega} \varphi\right)(t)= & { }^{\psi} D^{m, \omega \psi} \mathcal{I}_{a^{+}}^{m-\mu, \omega} \varphi(t) \\
= & \frac{\psi}{\omega^{m-\mu} \Gamma(m-\mu)} \int_{a}^{m, \omega} \exp \left[\frac{\omega-1}{\omega}(\psi(t)-\psi(u))\right] \\
& \times(\psi(t)-\psi(u))^{m-\mu-1} \psi^{\prime}(u) \varphi(u) d u
\end{aligned}
$$

and

$$
\begin{aligned}
\left({ }^{\psi} D_{b^{-}}^{\mu, \omega} \varphi\right)(t)= & { }_{\gamma}^{\psi} D^{m, \omega \psi} \mathcal{I}_{b^{-}}^{m-\mu, \omega} \varphi(t) \\
= & \frac{{ }_{\gamma} D_{t}^{m, \omega}}{\omega^{m-\mu} \Gamma(m-\mu)} \int_{t}^{b} \exp \left[\frac{\omega-1}{\omega}(\psi(u)-\psi(t))\right] \\
& \times(\psi(u)-\psi(t))^{m-\mu-1} \psi^{\prime}(u) \varphi(u) d u,
\end{aligned}
$$

where

$$
{ }^{\psi} D^{m, \omega}=\underbrace{\psi D^{\omega \psi} D^{\omega} \ldots \psi}_{m \text {-times }} D^{\omega}, \quad m=[\operatorname{Re}(\mu)]+1
$$

and

$$
\left({ }_{\gamma}^{\psi} D^{\omega} \varphi\right)(t)=(1-\omega) \varphi(t)-\omega \frac{\varphi^{\prime}(t)}{\psi^{\prime}(t)}, \quad{ }_{\gamma}^{\psi} D^{m, \omega}=\underbrace{{ }_{\gamma}^{\psi} D_{\gamma}^{\omega \psi} D^{\omega} \ldots{ }_{\gamma}^{\psi} D^{\omega}}_{m \text {-times }} .
$$

The functions $\left({ }^{\psi} D_{a^{+}}^{\mu, \omega} \varphi\right)(t)$ and $\left({ }^{\psi} D_{b^{-}}^{\mu, \omega} \varphi\right)(t)$ are called, respectively, the left- and right-sided proportional fractional derivatives of the function $\varphi$ with respect to the function $\psi$ for the order $\mu$.

Definition 2.7 ([36]) For an integrable function $\varphi$, a strictly increasing continuous function $\psi$ on $[a, b]$, and $\omega \in(0,1]$, we have, for all $\mu \in \mathbb{C}, \operatorname{Re}(\mu) \geq 0$,

$$
\begin{aligned}
& \left({ }^{\psi} \mathcal{I}_{a^{+}}^{\mu, \omega} \varphi\right)(t) \\
& \quad=\frac{1}{\omega^{\mu} \Gamma(\mu)} \int_{a}^{t} \exp \left[\frac{\omega-1}{\omega}(\psi(t)-\psi(u))\right](\psi(t)-\psi(u))^{\mu-1} \psi^{\prime}(u) \varphi(u) d u
\end{aligned}
$$

and

$$
\begin{aligned}
& \left({ }^{\psi} \mathcal{I}_{b^{-}}^{\mu, \omega} \varphi\right)(t) \\
& \quad=\frac{1}{\omega^{\mu} \Gamma(\mu)} \int_{t}^{b} \exp \left[\frac{\omega-1}{\omega}(\psi(u)-\psi(t))\right](\psi(u)-\psi(t))^{\mu-1} \psi^{\prime}(u) \varphi(u) d u .
\end{aligned}
$$


The functions $\left({ }^{\psi} \mathcal{I}_{a^{+}}^{\mu, \omega} \varphi\right)(t)$ and $\left({ }^{\psi} \mathcal{I}_{b^{-}}^{\mu, \omega} \varphi\right)(t)$ are called, respectively, the left- and right-sided proportional fractional integrals of the function $\varphi$ with respect to the function $\psi$ for the order $\mu$.

Lemma 2.1 ([36]) Let $\omega \in(0,1], \operatorname{Re}(\alpha)>0$ and $\operatorname{Re}(\mu)>0$. Then, if $\psi$ be continuous function and defined for $t \geq a$ or $t \leq b$, we have

$$
\begin{aligned}
& { }^{\psi} \mathcal{I}_{a^{+}}^{\mu, \omega}\left({ }^{\psi} \mathcal{I}_{a^{+}}^{\alpha, \omega} \varphi\right)(t)={ }^{\psi} \mathcal{I}_{a^{+}}^{\alpha, \omega}\left({ }^{\psi} \mathcal{I}_{a^{+}}^{\mu, \omega} \varphi\right)(t)=\left({ }^{\psi} \mathcal{I}_{a^{+}}^{\mu+\alpha, \omega} \varphi\right)(t), \\
& { }^{\psi} \mathcal{I}_{b^{-}}^{\mu, \omega}\left({ }^{\psi} \mathcal{I}_{b^{-}}^{\alpha, \omega} \varphi\right)(t)={ }^{\psi} \mathcal{I}_{b^{-}}^{\mu, v}\left({ }^{\psi} \mathcal{I}_{b^{-}}^{\alpha, \omega} \varphi\right)(t)=\left({ }^{\psi} \mathcal{I}_{b^{-}}^{\mu+\alpha, \omega} \varphi\right)(t) .
\end{aligned}
$$

Lemma $2.2([36])$ Let $0 \leq m<[\operatorname{Re}(\mu)]+1$. Then, we have

$$
\begin{aligned}
& { }^{\psi} D^{m, \omega}\left({ }^{\psi} \mathcal{I}_{a^{+}}^{\mu, \omega} \varphi\right)(t)=\left({ }^{\psi} \mathcal{I}_{a^{+}}^{\mu-m, \omega} \varphi\right)(t), \\
& { }_{\gamma}^{\psi} D^{m, \omega}\left({ }^{\psi} \mathcal{I}_{b^{-}}^{\mu, \omega} \varphi\right)(t)=\left({ }^{\psi} \mathcal{I}_{b^{-}}^{\mu-m, \omega} \varphi\right)(t) .
\end{aligned}
$$

Along this paper, we need the following identity from [37]:

Let $\omega \in(0,1], \mu \in \mathbb{C}, \operatorname{Re}(\mu) \geq 0$, and let $\psi$ be a strictly increasing continuous function. Then for any constant $k$, we have

$$
\left({ }^{\psi} \mathcal{I}_{a^{+}}^{\mu, \omega} k\right)(b)=\frac{(\psi(b)-\psi(a))^{\mu}}{\omega^{\mu} \Gamma(\mu+1)} k
$$

\section{Proportional fractional integral Hermite-Hadamard's inequalities concerning the function $\psi$}

The first part of our main contributions is within this section. Here we give HermiteHadamard's inequalities involving a convex function for proportional fractional integral operators concerning another strictly increasing continuous function $\psi$.

Theorem 3.1 Let $\psi: \digamma \rightarrow[a, b] \subseteq \mathbb{R}$ with $0 \leq a<b$ be a strictly increasing continuous function, and let $\varphi:[a, b] \rightarrow \mathbb{R}$ be a differentiable convex function on $(a, b)$ such that $(\varphi \circ$ $\psi): \digamma \rightarrow \mathbb{R}$ is an integrable function on $\digamma$. Then we have the following inequalities:

$$
\begin{aligned}
\varphi\left(\frac{a+b}{2}\right) & \leq \frac{\omega^{\mu} \Gamma(\mu+1)}{2(b-a)^{\mu}}\left\{{ }^{\psi} \mathcal{I}_{\left\{\psi^{-1}(a)\right\}^{+}}^{\mu, \omega}(\varphi \circ \psi)\left(\psi^{-1}(b)\right)+{ }^{\psi} \mathcal{I}_{\left\{\psi^{-1}(b)\right\}^{-}}^{\mu, \omega}(\varphi \circ \psi)\left(\psi^{-1}(a)\right)\right\} \\
& \leq \frac{\varphi(a)+\varphi(b)}{2} .
\end{aligned}
$$

Proof By the convexity of $\varphi$, for any $r, s \in[a, b]$, we can write

$$
\varphi(\lambda r+(1-\lambda) s) \leq \lambda \varphi(r)+(1-\lambda) \varphi(s)
$$

Putting $\lambda=\frac{1}{2}$, we have

$$
\varphi\left(\frac{r+s}{2}\right) \leq \frac{\varphi(r)+\varphi(s)}{2} .
$$

Let

$$
r=\eta a+(1-\eta) b
$$


and

$$
s=(1-\eta) a+\eta b .
$$

Substituting (3.4) and (3.5) into (3.3), we obtain

$$
2 \varphi\left(\frac{a+b}{2}\right) \leq \varphi\{\eta a+(1-\eta) b\}+\varphi\{(1-\eta) a+\eta b\} .
$$

Multiplying both sides of (3.6) by $\exp \left[\frac{\omega-1}{\omega} \eta(b-a)\right] \eta^{\mu-1}$ and then integrating the resulting inequality with respect to $\eta$ over $[0,1]$, we obtain

$$
\begin{aligned}
2 \varphi\left(\frac{a+b}{2}\right) \int_{0}^{1} \exp \left[\frac{\omega-1}{\omega} \eta(b-a)\right] \eta^{\mu-1} d \eta \\
\leq \int_{0}^{1} \exp \left[\frac{\omega-1}{\omega} \eta(b-a)\right] \eta^{\mu-1} \varphi\{\eta a+(1-\eta) b\} d \eta \\
\quad+\int_{0}^{1} \exp \left[\frac{\omega-1}{\omega} \eta(b-a)\right] \eta^{\mu-1} \varphi\{(1-\eta) a+\eta b\} d \eta .
\end{aligned}
$$

Substituting $\eta=\frac{b-\psi(u)}{b-a}$ into to the left-hand side of (3.7) and employing the fact that ${ }_{a}^{\psi} \mathcal{I}^{\mu, \omega}(1)=\frac{(\psi(b)-\psi(a))^{\mu}}{\omega^{\mu} \Gamma(\mu+1)}$, we obtain

$$
\begin{aligned}
\frac{2}{\mu} \varphi\left(\frac{a+b}{2}\right) \\
\leq \int_{0}^{1} \exp \left[\frac{\omega-1}{\omega} \eta(b-a)\right] \eta^{\mu-1} \varphi\{\eta a+(1-\eta) b\} d \eta \\
\quad+\int_{0}^{1} \exp \left[\frac{\omega-1}{\omega} \eta(b-a)\right] \eta^{\mu-1} \varphi\{(1-\eta) a+\eta b\} d \eta
\end{aligned}
$$

Next,

$$
\begin{aligned}
& \frac{\omega^{\mu} \Gamma(\mu+1)}{2(b-a)^{\mu}}\left\{{ }^{\psi} \mathcal{I}_{\left\{\psi^{-1}(a)\right\}^{+}}^{\mu, \omega}(\varphi \circ \psi)\left(\psi^{-1}(b)\right)+{ }^{\psi} \mathcal{I}_{\left\{\psi^{-1}(b)\right\}^{-}}^{\mu, \omega}(\varphi \circ \psi)\left(\psi^{-1}(a)\right)\right\} \\
& =\frac{\mu}{2(b-a)^{\mu}}\left\{\int_{\psi^{-1}(a)}^{\psi^{-1}(b)} \exp \left[\frac{\omega-1}{\omega}(b-\psi(u))\right](b-\psi(u))^{\mu-1} \varphi\{\psi(u)\} \psi^{\prime}(u) d u\right. \\
& \left.\quad+\int_{\psi^{-1}(a)}^{\psi^{-1}(b)} \exp \left[\frac{\omega-1}{\omega}(\psi(v)-a)\right](\psi(v)-a)^{\mu-1} \varphi\{\psi(v)\} \psi^{\prime}(v) d v\right\} \\
& =\frac{\mu}{2}\left\{\int_{\psi^{-1}(a)}^{\psi^{-1}(b)} \exp \left[\frac{\omega-1}{\omega}(b-\psi(u))\right]\left(\frac{b-\psi(u)}{b-a}\right)^{\mu-1} \varphi\{\psi(u)\} \frac{\psi^{\prime}(u)}{b-a} d u\right. \\
& \left.\quad+\int_{\psi^{-1}(a)}^{\psi^{-1}(b)} \exp \left[\frac{\omega-1}{\omega}(\psi(v)-a)\right]\left(\frac{\psi(v)-a}{b-a}\right)^{\mu-1} \varphi\{\psi(v)\} \frac{\psi^{\prime}(v)}{b-a} d v\right\} .
\end{aligned}
$$


Putting $\psi(u)=\eta a+(1-\eta) b$ and $\psi(v)=(1-\eta) a+\eta b$, we obtain

$$
\begin{aligned}
& \frac{\omega^{\mu} \Gamma(\mu+1)}{2(b-a)^{\mu}}\left\{{ }^{\psi} \mathcal{I}_{\left\{\psi^{-1}(a)\right\}^{+}}^{\mu, \omega}(\varphi \circ \psi)\left(\psi^{-1}(b)\right)+{ }^{\psi} \mathcal{I}_{\left\{\psi^{-1}(b)\right\}^{-}}^{\mu, \omega}(\varphi \circ \psi)\left(\psi^{-1}(a)\right)\right\} \\
& =\frac{\mu}{2}\left\{\int_{0}^{1} \exp \left[\frac{\omega-1}{\omega} \eta(b-a)\right] \eta^{\mu-1} \varphi\{\eta a+(1-\eta) b\} d \eta\right. \\
& \left.\quad+\int_{0}^{1} \exp \left[\frac{\omega-1}{\omega} \eta(b-a)\right] \eta^{\mu-1} \varphi\{(1-\eta) a+\eta b\} d \eta\right\} \\
& \geq \varphi\left(\frac{a+b}{2}\right) .
\end{aligned}
$$

So the first inequality in (3.1) is proved. To prove the second inequality, by the convexity of $\varphi$ we have

$$
\varphi(\eta a+(1-\eta) b) \leq \eta \varphi(a)+(1-\eta) \varphi(b)
$$

and

$$
\varphi((1-\eta) a+\eta b) \leq(1-\eta) \varphi(a)+\eta \varphi(b)
$$

Adding (3.10) and (3.11), we obtain

$$
\begin{aligned}
& \varphi(\eta a+(1-\eta) b)+\varphi((1-\eta) a+\eta b) \\
& \quad \leq \varphi(a)+\varphi(b) .
\end{aligned}
$$

Now multiplying both sides of (3.12) by $\exp \left[\frac{\omega-1}{\omega} \eta(b-a)\right] \eta^{\mu-1}$ and then integrating the resulting inequality with respect to $\eta$ over $[0,1]$, we obtain

$$
\begin{aligned}
& \int_{0}^{1} \exp \left[\frac{\omega-1}{\omega} \eta(b-a)\right] \eta^{\mu-1} \varphi(\eta a+(1-\eta) b) d \eta \\
& \quad+\int_{0}^{1} \exp \left[\frac{\omega-1}{\omega} \eta(b-a)\right] \eta^{\mu-1} \varphi((1-\eta) a+\eta b) d \eta \\
& \leq \frac{\varphi(a)+\varphi(b)}{\mu}
\end{aligned}
$$

So we have

$$
\begin{aligned}
& \frac{\omega^{\mu} \Gamma(\mu)}{(b-a)^{\mu}}\left\{{ }^{\psi} \mathcal{I}_{\left\{\psi^{-1}(a)\right\}^{+}}^{\mu, \omega}(\varphi \circ \psi)\left(\psi^{-1}(b)\right)+{ }^{\psi} \mathcal{I}_{\left\{\psi^{-1}(b)\right\}^{-}}^{\mu, \omega}(\varphi \circ \psi)\left(\psi^{-1}(a)\right)\right\} \\
& \quad \leq \frac{\varphi(a)+\varphi(b)}{\mu}
\end{aligned}
$$

Hence, in view of inequalities (3.9) and (3.13), we get the desired inequality (3.1).

\section{Remark 3.1}

(i) If we put $\omega=1$, then Theorem 3.1 reduces to Theorem 1.4 proved by Liu [19] in 2019 for the $\psi$-Riemann-Liouville fractional integral. 
(ii) If we put $\omega=1, \psi(0)=0$, and $\psi(1)=1$, and if $\varphi$ is a concave function, then both inequalities (3.1) are reversed, and Theorem 3.1 reduces to Theorem 6 obtained by Aljaaidi and Pachpatte [38] in 2020 for the $\psi$-Riemann-Liouville fractional integral.

(iii) If we put $\omega=1$ and $\psi(r)=r, r \in[a, b]$, then Theorem 3.1 reduces to Theorem 1.2 proved by Sarikaya et al. [17] in 2013 for the classical Riemann-Liouville fractional integral.

(iv) Applying inequalities (3.1) for $\psi(r)=r, r \in[a, b]$, and $\omega=1, \mu=1$, we obtain the classical Hermite-Hadamard inequalities for the classical integral (1.1).

Theorem 3.2 Let $\psi: \digamma \rightarrow[a, b] \subseteq \mathbb{R}$ with $0 \leq a<b$ be a strictly increasing continuous function, and let $\varphi:[a, b] \rightarrow \mathbb{R}$ be a differentiable convex function on $(a, b)$ such that $(\varphi \circ$ $\psi): \digamma \rightarrow \mathbb{R}$ is an integrable function on $\digamma$. Then we have the following inequalities:

$$
\begin{aligned}
& \varphi\left(\frac{a+b}{2}\right) \\
& \quad \leq \frac{\omega^{\mu} \Gamma(\mu+1)}{2(b-a)^{\mu}}\left\{{ }^{\psi} \mathcal{I}_{\left\{\psi^{-1}\left(\frac{a+b}{2}\right)\right\}^{-}}^{\mu, \omega}(\varphi \circ)\left(\psi^{-1}(a)\right)+{ }^{\psi} \mathcal{I}_{\left\{\psi^{-1}\left(\frac{a+b}{2}\right)\right\}^{+}}^{\mu, \omega}(\varphi \circ \psi)\left(\psi^{-1}(b)\right)\right\} \\
& \quad \leq \frac{\varphi(a)+\varphi(b)}{2}
\end{aligned}
$$

Proof By the convexity of the function $\varphi$, for all $r, s \in[a, b]$, we have

$$
\varphi\left(\frac{r+s}{2}\right) \leq \frac{\varphi(r)+\varphi(s)}{2}
$$

Putting $r=\frac{\eta}{2} b+\frac{2-\eta}{2} a$ and $s=\frac{2-\eta}{2} b+\frac{\eta}{2} a$, it follows that for all $r, s \in[a, b]$ and $\eta \in[0,1]$,

$$
\varphi\left(\frac{a+b}{2}\right) \leq \frac{1}{2}\left\{\varphi\left(\frac{\eta}{2} b+\frac{2-\eta}{2} a\right)+\varphi\left(\frac{2-\eta}{2} b+\frac{\eta}{2} a\right)\right\} .
$$

Multiplying both sides of (3.15) by $\exp \left[\frac{\omega-1}{\omega} \frac{\eta}{2}(b-a)\right]\left(\frac{\eta}{2}\right)^{\mu-1}$ and then integrating the resulting inequality with respect to $\eta$ over $[0,1]$, we obtain

$$
\begin{aligned}
\frac{1}{\mu} \varphi\left(\frac{a+b}{2}\right) \\
\leq \int_{0}^{1} \exp \left[\frac{\omega-1}{\omega} \frac{\eta}{2}(b-a)\right]\left(\frac{\eta}{2}\right)^{\mu-1} \varphi\left(\frac{\eta}{2} b+\frac{2-\eta}{2} a\right) d \eta \\
\quad+\int_{0}^{1} \exp \left[\frac{\omega-1}{\omega} \frac{\eta}{2}(b-a)\right]\left(\frac{\eta}{2}\right)^{\mu-1} \varphi\left(\frac{2-\eta}{2} b+\frac{\eta}{2} a\right) d \eta
\end{aligned}
$$

Next,

$$
\begin{aligned}
& \frac{\omega^{\mu} \Gamma(\mu+1)}{2(b-a)^{\mu}}\left\{{ }^{\psi} \mathcal{I}_{\left\{\psi^{-1}\left(\frac{a+b}{2}\right)\right\}^{-}}^{\mu, \omega}(\varphi \circ \psi)\left(\psi^{-1}(a)\right)+{ }^{\psi} \mathcal{I}_{\left\{\psi^{-1}\left(\frac{a+b}{2}\right)\right\}^{+}}^{\mu, \omega}(\varphi \circ \psi)\left(\psi^{-1}(b)\right)\right\} \\
& =\frac{\mu}{2(b-a)^{\mu}}\left\{\int_{\psi^{-1}(a)}^{\psi^{-1}\left(\frac{a+b}{2}\right)} \exp \left[\frac{\omega-1}{\omega}(\psi(u)-a)\right](\psi(u)-a)^{\mu-1}(\varphi \circ \psi)(u) \psi^{\prime}(u) d u\right. \\
& \left.\quad+\int_{\psi^{-1}\left(\frac{a+b}{2}\right)}^{\psi^{-1}(b)} \exp \left[\frac{\omega-1}{\omega}(b-\psi(v))\right](b-\psi(v))^{\mu-1}(\varphi \circ \psi)(v) \psi^{\prime}(v) d v\right\}
\end{aligned}
$$




$$
\begin{aligned}
= & \mu\left\{\int_{\psi^{-1}(a)}^{\psi^{-1}\left(\frac{a+b}{2}\right)} \exp \left[\frac{\omega-1}{\omega}(\psi(u)-a)\right]\left(\frac{\psi(u)-a}{b-a}\right)^{\mu-1}(\varphi \circ \psi)(u) \frac{\psi^{\prime}(u)}{b-a} d u\right. \\
& \left.+\int_{\psi^{-1}\left(\frac{a+b}{2}\right)}^{\psi^{-1}(b)} \exp \left[\frac{\omega-1}{\omega}(b-\psi(v))\right]\left(\frac{b-\psi(v)}{b-a}\right)^{\mu-1}(\varphi \circ \psi)(v) \frac{\psi^{\prime}(v)}{b-a} d v\right\}
\end{aligned}
$$

Putting $\psi(u)=\frac{\eta}{2} b+\frac{2-\eta}{2} a$ and $\psi(v)=\frac{2-\eta}{2} b+\frac{\eta}{2} a$, we obtain

$$
\begin{aligned}
& \frac{\omega^{\mu} \Gamma(\mu+1)}{2(b-a)^{\mu}}\left\{{ }^{\psi} \mathcal{I}_{\left\{\psi^{-1}\left(\frac{a+b}{2}\right)\right\}^{-}}^{\mu, \omega}(\varphi \circ \psi)\left(\psi^{-1}(a)\right)+{ }^{\psi} \mathcal{I}_{\left\{\psi^{-1}\left(\frac{a+b}{2}\right)\right\}^{+}}^{\mu, \omega}(\varphi)\left(\psi^{-1}(b)\right)\right\} \\
& =\mu\left\{\int_{0}^{1} \exp \left[\frac{\omega-1}{\omega} \frac{\eta}{2}(b-a)\right]\left(\frac{\eta}{2}\right)^{\mu-1} \varphi\left(\frac{\eta}{2} b+\frac{2-\eta}{2} a\right) d \eta\right. \\
& \left.\quad+\int_{0}^{1} \exp \left[\frac{\omega-1}{\omega} \frac{\eta}{2}(b-a)\right]\left(\frac{\eta}{2}\right)^{\mu-1} \varphi\left(\frac{2-\eta}{2} b+\frac{\eta}{2} a\right) d \eta\right\} \\
& \geq \varphi\left(\frac{a+b}{2}\right)
\end{aligned}
$$

which proves the first inequality in (3.14). To prove the second inequality, using the the convexity of the function $\varphi$, we have

$$
\begin{aligned}
\varphi\left(\frac{\eta}{2} b+\frac{2-\eta}{2} a\right) & \leq \frac{\eta}{2} \varphi(b)+\frac{2-\eta}{2} \varphi(a), \\
\varphi\left(\frac{2-\eta}{2} b+\frac{\eta}{2} a\right) & \leq\left(\frac{2-\eta}{2} \varphi(b)+\frac{\eta}{2} \varphi(a)\right) .
\end{aligned}
$$

Adding inequalities (3.18) and (3.19), we get

$$
\varphi\left(\frac{\eta}{2} b+\frac{2-\eta}{2} a\right)+\varphi\left(\frac{2-\eta}{2} b+\frac{\eta}{2} a\right) \leq \varphi(a)+\varphi(b) .
$$

Multiplying both sides of (3.20) by $\exp \left[\frac{\omega-1}{\omega} \frac{\eta}{2}(b-a)\right]\left(\frac{\eta}{2}\right)^{\mu-1}$ and then integrating the resulting inequality with respect to $\eta$ over $[0,1]$, we obtain

$$
\begin{aligned}
& \mu\left\{\int_{0}^{1} \exp \left[\frac{\omega-1}{\omega} \frac{\eta}{2}(b-a)\right]\left(\frac{\eta}{2}\right)^{\mu-1} \varphi\left(\frac{\eta}{2} b+\frac{2-\eta}{2} a\right) d \eta\right. \\
& \left.\quad+\int_{0}^{1} \exp \left[\frac{\omega-1}{\omega} \frac{\eta}{2}(b-a)\right]\left(\frac{\eta}{2}\right)^{\mu-1} \varphi\left(\frac{2-\eta}{2} b+\frac{\eta}{2} a\right) d \eta\right\} \\
& \leq \varphi(a)+\varphi(b) .
\end{aligned}
$$

By comparing the left-hand-side of inequality (3.17) with the left-hand-side of inequality (3.21) we can deduce

$$
\begin{aligned}
& \frac{\omega^{\mu} \Gamma(\mu+1)}{2(b-a)^{\mu}}\left\{{ }^{\psi} \mathcal{I}_{\left\{\psi^{-1}\left(\frac{a+b}{2}\right)\right\}^{-}}^{\mu, \omega}(\varphi \circ)\left(\psi^{-1}(a)\right)+{ }^{\psi} \mathcal{I}_{\left\{\psi^{-1}\left(\frac{a+b}{2}\right)\right\}^{+}}^{\mu, \omega}(\varphi \circ \psi)\left(\psi^{-1}(b)\right)\right\} \\
& \quad \leq \frac{\varphi(a)+\varphi(b)}{2}
\end{aligned}
$$

which is the second inequality in (3.14). The proof is thus completed. 


\section{Proportional fractional integral Hermite-Hadamard-type inequalities concerning the function $\psi$}

This section is devoted to the second part of our contributions, which involve a generalization of some Hermite-Hadamard-type inequalities introduced by Chen via the RiemannLiouville fractional integral. For the current generalization, we use the fractional integral [36] introduced by Jarad (2020).

Theorem 4.1 Let $\psi:[a, b] \rightarrow \digamma \subseteq \mathbb{R}$, with $0 \leq a<b$ be a strictly increasing function, and let $\varphi: \digamma \rightarrow \mathbb{R}$ be a positive twice differentiable function on $\digamma^{\circ}$ such that $(\varphi \circ \psi):[a, b] \rightarrow \mathbb{R}$ is an integrable function on $[a, b]$. If $\varphi^{\prime \prime}$ is bounded in $[a, b]$, then, we have the following inequalities:

$$
\begin{aligned}
& \frac{\mu C_{1}}{2(b-a)^{\mu}} \int_{\psi^{-1}(a)}^{\psi^{-1}\left(\frac{a+b}{2}\right)}\left(\frac{a+b}{2}-\psi(u)\right)^{2}\left\{\exp \left[\frac{\omega-1}{\omega}(b-\psi(u))\right](b-\psi(u))^{\mu-1}\right. \\
& \left.\quad+\exp \left[\frac{\omega-1}{\omega}(\psi(u)-a)\right](\psi(u)-a)^{\mu-1}\right\} \psi^{\prime}(u) d u \\
& \leq \frac{\omega^{\mu} \Gamma(\mu+1)}{2(b-a)^{\mu}}\left\{{ }^{\psi} \mathcal{I}_{\left\{\psi^{-1}(a)\right\}^{+}}^{\mu, \omega}(\varphi \circ \psi)\left(\psi^{-1}(b)\right)+{ }^{\psi} \mathcal{I}_{\left\{\psi^{-1}(b)\right\}^{-}}^{\mu, \omega}(\varphi \circ \psi)\left(\psi^{-1}(a)\right)\right\} \\
& \quad-\varphi\left\{\frac{a+b}{2}\right\} \\
& \leq \frac{\mu C_{2}}{2(b-a)^{\mu}} \int_{\psi^{-1}(a)}^{\psi^{-1}\left(\frac{a+b}{2}\right)}\left(\frac{a+b}{2}-\psi(u)\right)^{2}\left\{\exp \left[\frac{\omega-1}{\omega}(b-\psi(u))\right](b-\psi(u))^{\mu-1}\right. \\
& \left.\quad+\exp \left[\frac{\omega-1}{\omega}(\psi(u)-a)\right](\psi(u)-a)^{\mu-1}\right\} \psi^{\prime}(u) d u .
\end{aligned}
$$

Proof We have

$$
\begin{aligned}
& \frac{\omega^{\mu} \Gamma(\mu+1)}{2(b-a)^{\mu}}\left\{{ }^{\psi} \mathcal{I}_{\left\{\psi^{-1}(a)\right\}^{+}}^{\mu, \omega}(\varphi \circ \psi)\left(\psi^{-1}(b)\right)+{ }^{\psi} \mathcal{I}_{\left\{\psi^{-1}(b)\right\}^{-}}^{\mu, \omega}(\varphi \circ \psi)\left(\psi^{-1}(a)\right)\right\} \\
&=\frac{\mu}{2(b-a)^{\mu}}\left\{\int_{\psi^{-1}(a)}^{\psi^{-1}(b)} \exp \left[\frac{\omega-1}{\omega}(b-\psi(u))\right](b-\psi(u))^{\mu-1} \varphi\{\psi(u)\} \psi^{\prime}(u) d u\right. \\
&\left.+\int_{\psi^{-1}(a)}^{\psi^{-1}(b)} \exp \left[\frac{\omega-1}{\omega}(\psi(u)-a)\right](\psi(u)-a)^{\mu-1} \varphi\{\psi(u)\} \psi^{\prime}(u) d u\right\} \\
&= \frac{\mu}{2(b-a)^{\mu}} \int_{\psi^{-1}(a)}^{\psi^{-1}(b)}\left\{\exp \left[\frac{\omega-1}{\omega}(b-\psi(u))\right](b-\psi(u))^{\mu-1}\right. \\
&\left.+\exp \left[\frac{\omega-1}{\omega}(\psi(u)-a)\right](\psi(u)-a)^{\mu-1}\right\} \varphi\{\psi(u)\} \psi^{\prime}(u) d u \\
&= \frac{\mu}{2(b-a)^{\mu}} \int_{\psi^{-1}(a)}^{\psi^{-1}(b)}\left\{\exp \left[\frac{\omega-1}{\omega}(b-\psi(u))\right](b-\psi(u))^{\mu-1}\right. \\
&\left.+\exp \left[\frac{\omega-1}{\omega}(\psi(u)-a)\right](\psi(u)-a)^{\mu-1}\right\} \varphi\{a+b-\psi(u)\} \psi^{\prime}(u) d u .
\end{aligned}
$$

So we can write

$$
\frac{\omega^{\mu} \Gamma(\mu+1)}{2(b-a)^{\mu}}\left\{{ }^{\psi} \mathcal{I}_{\left\{\psi^{-1}(a)\right\}^{+}}^{\mu, \omega}(\varphi \circ \psi)\left(\psi^{-1}(b)\right)+{ }^{\psi} \mathcal{I}_{\left\{\psi^{-1}(b)\right\}^{-}}^{\mu, \omega}(\varphi \circ \psi)\left(\psi^{-1}(a)\right)\right\}
$$


Aljaaidi et al. Advances in Difference Equations

(2021) 2021:493

Page 13 of 19

$$
\begin{aligned}
= & \frac{\mu}{4(b-a)^{\mu}} \int_{\psi^{-1}(a)}^{\psi^{-1}(b)}\left\{\exp \left[\frac{\omega-1}{\omega}(b-\psi(u))\right](b-\psi(u))^{\mu-1}\right. \\
& \left.+\exp \left[\frac{\omega-1}{\omega}(\psi(u)-a)\right](\psi(u)-a)^{\mu-1}\right\}(\varphi\{\psi(u)\}+\varphi\{a+b-\psi(u)\}) \psi^{\prime}(u) d u,
\end{aligned}
$$

which we can rewrite as

$$
\begin{aligned}
& \frac{\omega^{\mu} \Gamma(\mu+1)}{2(b-a)^{\mu}}\left\{{ }^{\psi} \mathcal{I}_{\left\{\psi^{-1}(a)\right\}^{+}}^{\mu, \omega}(\varphi \circ \psi)\left(\psi^{-1}(b)\right)+{ }^{\psi} \mathcal{I}_{\left\{\psi^{-1}(b)\right\}^{-}}^{\mu, \omega}(\varphi \circ \psi)\left(\psi^{-1}(a)\right)\right\}-\varphi\left\{\frac{a+b}{2}\right\} \\
& =\frac{\mu}{4(b-a)^{\mu}} \int_{\psi^{-1}(a)}^{\psi^{-1}(b)}\left\{\exp \left[\frac{\omega-1}{\omega}(b-\psi(u))\right](b-\psi(u))^{\mu-1}\right. \\
& \left.\quad+\exp \left[\frac{\omega-1}{\omega}(\psi(u)-a)\right](\psi(u)-a)^{\mu-1}\right\} \\
& \quad \times\left(\varphi\{a+b-\psi(u)\}+\varphi\{\psi(u)\}-2 \varphi\left\{\frac{a+b}{2}\right\}\right) \psi^{\prime}(u) d u .
\end{aligned}
$$

Since

$$
\begin{aligned}
& \left\{\exp \left[\frac{\omega-1}{\omega}(b-\psi(u))\right](b-\psi(u))^{\mu-1}+\exp \left[\frac{\omega-1}{\omega}(\psi(u)-a)\right](\psi(u)-a)^{\mu-1}\right\} \\
& \quad \times\left(\varphi\{a+b-\psi(u)\}+\varphi\{\psi(u)\}-2 \varphi\left\{\frac{a+b}{2}\right\}\right)
\end{aligned}
$$

is symmetric about $u=\psi^{-1}\left(\frac{a+b}{2}\right)$, we have

$$
\begin{aligned}
& \frac{\mu}{4(b-a)^{\mu}} \int_{\psi^{-1}(a)}^{\psi^{-1}(b)}\left\{\exp \left[\frac{\omega-1}{\omega}(b-\psi(u))\right](b-\psi(u))^{\mu-1}\right. \\
& \left.\quad+\exp \left[\frac{\omega-1}{\omega}(\psi(u)-a)\right](\psi(u)-a)^{\mu-1}\right\} \\
& \quad \times\left(\varphi\{a+b-\psi(u)\}+\varphi\{\psi(u)\}-2 \varphi\left\{\frac{a+b}{2}\right\}\right) \psi^{\prime}(u) d u \\
& =\frac{\mu}{2(b-a)^{\mu}} \int_{\psi^{-1}(a)}^{\psi^{-1}\left(\frac{a+b}{2}\right)}\left\{\exp \left[\frac{\omega-1}{\omega}(b-\psi(u))\right](b-\psi(u))^{\mu-1}\right. \\
& \left.\quad+\exp \left[\frac{\omega-1}{\omega}(\psi(u)-a)\right](\psi(u)-a)^{\mu-1}\right\} \\
& \quad \times\left(\varphi\{a+b-\psi(u)\}+\varphi\{\psi(u)\}-2 \varphi\left\{\frac{a+b}{2}\right\}\right) \psi^{\prime}(u) d u
\end{aligned}
$$

which yields

$$
\begin{aligned}
& \frac{\omega^{\mu} \Gamma(\mu+1)}{2(b-a)^{\mu}}\left\{{ }^{\psi} \mathcal{I}_{\left\{\psi^{-1}(a)\right\}^{+}}^{\mu, \omega}(\varphi \circ \psi)\left(\psi^{-1}(b)\right)+{ }^{\psi} \mathcal{I}_{\left\{\psi^{-1}(b)\right\}^{-}}^{\mu, \omega}(\varphi \circ \psi)\left(\psi^{-1}(a)\right)\right\}-\varphi\left\{\frac{a+b}{2}\right\} \\
& =\frac{\mu}{2(b-a)^{\mu}} \int_{\psi^{-1}(a)}^{\psi^{-1}\left(\frac{a+b}{2}\right)}\left\{\exp \left[\frac{\omega-1}{\omega}(b-\psi(u))\right](b-\psi(u))^{\mu-1}\right. \\
& \left.\quad+\exp \left[\frac{\omega-1}{\omega}(\psi(u)-a)\right](\psi(u)-a)^{\mu-1}\right\}
\end{aligned}
$$




$$
\times\left(\varphi\{a+b-\psi(u)\}+\varphi\{\psi(u)\}-2 \varphi\left\{\frac{a+b}{2}\right\}\right) \psi^{\prime}(u) d u
$$

Since

$$
\varphi\{a+b-\psi(u)\}-\varphi\left\{\frac{a+b}{2}\right\}=\int_{\frac{a+b}{2}}^{a+b-\psi(u)} \varphi^{\prime}\{\psi(t)\} d\{\psi(t)\}
$$

and

$$
\varphi\left\{\frac{a+b}{2}\right\}-\varphi\{\psi(u)\}=\int_{\psi(u)}^{\frac{a+b}{2}} \varphi^{\prime}\{\psi(t)\} d\{\psi(t)\}
$$

we have

$$
\begin{gathered}
\varphi\{\psi(u)\}+\varphi\{a+b-\psi(u)\}-2 \varphi\left\{\frac{a+b}{2}\right\} \\
=\int_{\frac{a+b}{2}}^{a+b-\psi(u)} \varphi^{\prime}\{\psi(t)\} d\{\psi(t)\}-\int_{\psi(u)}^{\frac{a+b}{2}} \varphi^{\prime}\{\psi(t)\} d\{\psi(t)\} \\
=\int_{\psi(u)}^{\frac{a+b}{2}} \varphi^{\prime}\{a+b-\psi(t)\} d\{\psi(t)\}-\int_{\psi(u)}^{\frac{a+b}{2}} \varphi^{\prime}\{\psi(t)\} d\{\psi(t)\} \\
=\int_{\psi(u)}^{\frac{a+b}{2}}\left[\varphi^{\prime}\{a+b-\psi(t)\}-\varphi^{\prime}\{\psi(t)\}\right] d\{\psi(t)\} .
\end{gathered}
$$

Since

$$
\varphi^{\prime}\{a+b-\psi(t)\}-\varphi^{\prime}\{\psi(t)\}=\int_{\psi(t)}^{a+b-\psi(t)} \varphi^{\prime \prime}\{\psi(y)\} d\{\psi(y)\},
$$

for $\psi(t) \in\left[a, \frac{a+b}{2}\right]$, where $t \in \digamma$, we have

$$
C_{1}(a+b-2 \psi(t)) \leq \varphi^{\prime}\{a+b-\psi(t)\}-\varphi^{\prime}\{\psi(t)\} \leq C_{2}(a+b-2 \psi(t)),
$$

which leads to

$$
\begin{aligned}
C_{1}\left(\frac{a+b}{2}-\psi(u)\right)^{2} & \leq \int_{\psi(u)}^{\frac{a+b}{2}}\left[\varphi^{\prime}\{a+b-\psi(t)\}-\varphi^{\prime}\{\psi(t)\}\right] d\{\psi(t)\} \\
& \leq C_{2}\left(\frac{a+b}{2}-\psi(u)\right)^{2}
\end{aligned}
$$

that is,

$$
\begin{aligned}
C_{1}\left(\frac{a+b}{2}-\psi(u)\right)^{2} & \leq \varphi\{\psi(u)\}+\varphi\{a+b-\psi(u)\}-2 \varphi\left\{\frac{a+b}{2}\right\} \\
& \leq C_{2}\left(\frac{a+b}{2}-\psi(u)\right)^{2} .
\end{aligned}
$$

In view of inequality (4.6) and equality (4.4), we get the required inequality (4.1), which completes the proof. 
Theorem 4.2 Let $\psi:[a, b] \rightarrow \digamma \subseteq \mathbb{R}$, with $0 \leq a<b$ be a strictly increasing function, and let $\varphi: \digamma \rightarrow \mathbb{R}$ be a positive twice differentiable function on $\digamma^{\circ}$ such that $(\varphi \circ \psi):[a, b] \rightarrow \mathbb{R}$ is an integrable function on $[a, b]$. If $\varphi^{\prime \prime}$ is bounded in $[a, b]$, then we have the following inequalities:

$$
\begin{aligned}
& \frac{-\mu C_{2}}{2(b-a)^{\mu}} \int_{\psi^{-1}(a)}^{\left.\psi^{-1} \frac{(a+b}{2}\right)}[(b-\psi(u))(\psi(u)-a)]\left\{\exp \left[\frac{\omega-1}{\omega}(b-\psi(u))\right](b-\psi(u))^{\mu-1}\right. \\
& \left.\quad+\exp \left[\frac{\omega-1}{\omega}(\psi(u)-a)\right](\psi(u)-a)^{\mu-1}\right\} \psi^{\prime}(u) d u \\
& \leq \frac{\omega^{\mu} \Gamma(\mu+1)}{2(b-a)^{\mu}}\left\{{ }^{\psi} \mathcal{I}_{\left\{\psi^{-1}(a)\right\}^{+}}^{\mu, \omega}(\varphi \circ)\left(\psi^{-1}(b)\right)+{ }^{\psi} \mathcal{I}_{\left\{\psi^{-1}(b)\right\}^{-}}^{\mu, \omega}(\varphi \circ \psi)\left(\psi^{-1}(a)\right)\right\} \\
& \quad-\frac{\varphi(a)+\varphi(b)}{2} \\
& \leq \frac{-\mu C_{1}}{2(b-a)^{\mu}} \int_{\psi^{-1}(a)}^{\psi^{-1}\left(\frac{a+b}{2}\right)}[(b-\psi(u))(\psi(u)-a)] \\
& \quad \times\left\{\exp \left[\frac{\omega-1}{\omega}(b-\psi(u))\right](b-\psi(u))^{\mu-1}\right. \\
& \left.\quad+\exp \left[\frac{\omega-1}{\omega}(\psi(u)-a)\right](\psi(u)-a)^{\mu-1}\right\} \psi^{\prime}(u) d u .
\end{aligned}
$$

Proof In view of Theorem 4.1, using the inequality (4.2), we can write

$$
\begin{aligned}
& \frac{\omega^{\mu} \Gamma(\mu+1)}{2(b-a)^{\mu}}\left\{{ }^{\psi} \mathcal{I}_{\left\{\psi^{-1}(a)\right\}^{+}}^{\mu, \omega}(\varphi)\left(\psi^{-1}(b)\right)+{ }^{\psi} \mathcal{I}_{\left\{\psi^{-1}(b)\right\}^{-}}^{\mu, \omega}(\varphi \circ \psi)\left(\psi^{-1}(a)\right)\right\}-\frac{\varphi(a)+\varphi(b)}{2} \\
& =\frac{\mu}{4(b-a)^{\mu}} \int_{\psi^{-1}(a)}^{\psi^{-1}(b)}\left\{\exp \left[\frac{\omega-1}{\omega}(b-\psi(u))\right](b-\psi(u))^{\mu-1}\right. \\
& \left.\quad+\exp \left[\frac{\omega-1}{\omega}(\psi(u)-a)\right](\psi(u)-a)^{\mu-1}\right\} \\
& \quad \times(\varphi\{a+b-\psi(u)\}+\varphi\{\psi(u)\}-[\varphi(a)+\varphi(a)]) \psi^{\prime}(u) d u .
\end{aligned}
$$

Since

$$
\begin{aligned}
& \left\{\exp \left[\frac{\omega-1}{\omega}(b-\psi(u))\right](b-\psi(u))^{\mu-1}+\exp \left[\frac{\omega-1}{\omega}(\psi(u)-a)\right](\psi(u)-a)^{\mu-1}\right\} \\
& \quad \times(\varphi\{a+b-\psi(u)\}+\varphi\{\psi(u)\}-[\varphi(a)+\varphi(a)])
\end{aligned}
$$

is symmetric about $u=\psi^{-1}\left(\frac{a+b}{2}\right)$, we have

$$
\begin{aligned}
& \frac{\omega^{\mu} \Gamma(\mu+1)}{2(b-a)^{\mu}}\left\{{ }^{\psi} \mathcal{I}_{\left\{\psi^{-1}(a)\right\}^{+}}^{\mu, \omega}(\varphi \circ \psi)\left(\psi^{-1}(b)\right)+{ }^{\psi} \mathcal{I}_{\left\{\psi^{-1}(b)\right\}^{-}}^{\mu, \omega}(\varphi \circ \psi)\left(\psi^{-1}(a)\right)\right\}-\frac{\varphi(a)+\varphi(b)}{2} \\
& =\frac{\mu}{2(b-a)^{\mu}} \int_{\psi^{-1}(a)}^{\psi^{-1}\left(\frac{a+b}{2}\right)}\left\{\exp \left[\frac{\omega-1}{\omega}(b-\psi(u))\right](b-\psi(u))^{\mu-1}\right. \\
& \left.\quad+\exp \left[\frac{\omega-1}{\omega}(\psi(u)-a)\right](\psi(u)-a)^{\mu-1}\right\} \\
& \quad \times(\varphi\{a+b-\psi(u)\}+\varphi\{\psi(u)\}-[\varphi(a)+\varphi(b)]) \psi^{\prime}(u) d u .
\end{aligned}
$$


Since

$$
\varphi\{b\}-\varphi\{a+b-\psi(u)\}=\int_{a+b-\psi(u)}^{b} \varphi^{\prime}\{\psi(t)\} d\{\psi(t)\}
$$

and

$$
\varphi\{\psi(u)\}-\varphi\{a\}=\int_{a}^{\psi(u)} \varphi^{\prime}\{\psi(t)\} d\{\psi(t)\}
$$

we get

$$
\begin{aligned}
& \varphi\{\psi(u)\}+\varphi\{a+b-\psi(u)\}-[\varphi\{a\}+\varphi\{b\}] \\
& =\int_{a}^{\psi(u)} \varphi^{\prime}\{\psi(t)\} d\{\psi(t)\}-\int_{a+b-\psi(u)}^{b} \varphi^{\prime}\{\psi(t)\} d\{\psi(t)\} \\
& =\int_{a}^{\psi(u)} \varphi^{\prime}\{\psi(t)\} d\{\psi(t)\}-\int_{a}^{\psi(u)} \varphi^{\prime}\{a+b-\psi(t)\} d\{\psi(t)\} \\
& =-\int_{a}^{\psi(u)}\left[\varphi^{\prime}\{a+b-\psi(t)\}-\varphi^{\prime}\{\psi(t)\}\right] d\{\psi(t)\} .
\end{aligned}
$$

Since

$$
\varphi^{\prime}\{a+b-\psi(t)\}-\varphi^{\prime}\{\psi(t)\}=\int_{\psi(t)}^{a+b-\psi(t)} \varphi^{\prime \prime}\{\psi(y)\} d\{\psi(y)\}
$$

for $\psi(t) \in\left[a, \frac{a+b}{2}\right]$, where $t \in \digamma$, we have

$$
C_{1}(a+b-2 \psi(t)) \leq \varphi^{\prime}\{a+b-\psi(t)\}-\varphi^{\prime}\{\psi(t)\} \leq C_{2}(a+b-2 \psi(t))
$$

which leads to

$$
\begin{aligned}
-C_{2}[(b-\psi(u))(\psi(u)-a)] & \leq \int_{a}^{\psi(u)}\left[\varphi^{\prime}\{a+b-\psi(t)\}-\varphi^{\prime}\{\psi(t)\}\right] d\{\psi(t)\} \\
& \leq-C_{1}[(b-\psi(u))(\psi(u)-a)]
\end{aligned}
$$

that is,

$$
\begin{aligned}
-C_{2}[(b-\psi(u))(\psi(u)-a)] & \leq \varphi\{\psi(u)\}+\varphi\{a+b-\psi(u)\}-[\varphi\{a\}+\varphi\{b\}] \\
& \leq-C_{1}[(b-\psi(u))(\psi(u)-a)] .
\end{aligned}
$$

In view of inequality (4.10) and equality (4.8), we get the required inequality (4.7), which completes the proof.

Remark 4.1 If we put $\omega=1$ and $\psi(r)=r, r \in[a, b]$, then Theorems 4.1 and 4.2 reduce to Theorems 1.2 and 1.3, respectively, proved by Chen [18] in 2013 for classical RiemannLiouville fractional integral. 
The next result is reproof of inequality (3.3) without using the convexity of the function $\varphi$ by employing Theorems 4.1 and 4.2 .

Theorem 4.3 Let $\psi: \digamma \rightarrow[a, b] \subseteq \mathbb{R}$, with $0 \leq a<b$ be a strictly increasing continuous function, and let $\varphi:[a, b] \longrightarrow \mathbb{R}$ be a positive differentiable function on $(a, b)$ such that $(\varphi \circ \psi): \digamma \rightarrow \mathbb{R}$ is an integrable function on $\digamma$. If $\varphi^{\prime}\{a+b-\psi(u)\} \geq \varphi^{\prime}\{\psi(u)\}$ for all $\psi(u) \in$ $\left[a, \frac{a+b}{2}\right]$, then we have the following inequalities:

$$
\begin{aligned}
\varphi\left(\frac{a+b}{2}\right) & \leq \frac{\omega^{\mu} \Gamma(\mu+1)}{2(b-a)^{\mu}}\left\{{ }^{\psi} \mathcal{I}_{\left\{\psi^{-1}(a)\right\}^{+}}^{\mu, \omega}(\varphi \circ \psi)\left(\psi^{-1}(b)\right)+{ }^{\psi} \mathcal{I}_{\left\{\psi^{-1}(b)\right\}^{-}}^{\mu, \omega}(\varphi \circ \psi)\left(\psi^{-1}(a)\right)\right\} \\
& \leq \frac{\varphi(a)+\varphi(b)}{2} .
\end{aligned}
$$

Proof Using the assumption of Theorem 4.1, from (4.4) and (4.5) we have

$$
\begin{aligned}
& \frac{\omega^{\mu} \Gamma(\mu+1)}{2(b-a)^{\mu}}\left\{{ }^{\psi} \mathcal{I}_{\left\{\psi^{-1}(a)\right\}^{+}}^{\mu, \omega}(\varphi \circ \psi)\left(\psi^{-1}(b)\right)+{ }^{\psi} \mathcal{I}_{\left\{\psi^{-1}(b)\right\}^{-}}^{\mu, \omega}(\varphi \circ \psi)\left(\psi^{-1}(a)\right)\right\}-\varphi\left\{\frac{a+b}{2}\right\} \\
& =\frac{\mu}{2(b-a)^{\mu}} \int_{\psi^{-1}(a)}^{\psi^{-1}\left(\frac{a+b}{2}\right)}\left(\int_{\psi(u)}^{\frac{a+b}{2}}\left[\varphi^{\prime}\{a+b-\psi(t)\}-\varphi^{\prime}\{\psi(t)\}\right] d\{\psi(t)\}\right) \\
& \quad \times\left\{\exp \left[\frac{\omega-1}{\omega}(b-\psi(u))\right](b-\psi(u))^{\mu-1}\right. \\
& \left.\quad+\exp \left[\frac{\omega-1}{\omega}(\psi(u)-a)\right](\psi(u)-a)^{\mu-1}\right\} \psi^{\prime}(u) d u \geq 0 .
\end{aligned}
$$

Similarly, using the assumption of Theorem 4.2, from (4.8) and (4.9) we have

$$
\begin{aligned}
& \frac{\omega^{\mu} \Gamma(\mu+1)}{2(b-a)^{\mu}}\left\{{ }^{\psi} \mathcal{I}_{\left\{\psi^{-1}(a)\right\}^{+}}^{\mu, \omega}(\varphi)\left(\psi^{-1}(b)\right)+{ }^{\psi} \mathcal{I}_{\left\{\psi^{-1}(b)\right\}^{-}}^{\mu, \omega}(\varphi \circ \psi)\left(\psi^{-1}(a)\right)\right\}-\frac{\varphi(a)+\varphi(a)}{2} \\
& =\frac{\mu}{2(b-a)^{\mu}} \int_{\psi^{-1}(a)}^{\psi^{-1}\left(\frac{a+b}{2}\right)}\left(-\int_{a}^{\psi(u)}\left[\varphi^{\prime}\{a+b-\psi(t)\}-\varphi^{\prime}\{\psi(t)\}\right] d\{\psi(t)\}\right) \\
& \quad \times\left\{\exp \left[\frac{\omega-1}{\omega}(b-\psi(u))\right](b-\psi(u))^{\mu-1}\right. \\
& \left.\quad+\exp \left[\frac{\omega-1}{\omega}(\psi(u)-a)\right](\psi(u)-a)^{\mu-1}\right\} \psi^{\prime}(u) d u \leq 0 .
\end{aligned}
$$

This completes the proof.

\section{Conclusions}

Nowadays, we need to develop and refine our capabilities to generalize some recent results directly related to the topic of fractional calculus because this topic is interesting and has many applications in modeling natural phenomena in the world. Many researchers have generalized some different fractional operators using classical tools and operators in fractional calculus. One of these operators is the generalized proportional fractional integral. In this work, we have developed some new techniques of fractional integral HermiteHadamard inequalities concerning the generalized proportional fractional integral with 
respect to another strictly increasing continuous function. The current fractional integral has been employed to establish some new fractional integral Hermite-Hadamardtype inequalities. Furthermore, through our discussion of this work, we have succeeded in presenting some special cases related to the within results. In future work, we recommend researchers study the current inequality via recent fractional operators such as the Atangana-Baleanu operator [6] or Caputo-Fabrizio operator [39].

\author{
Acknowledgements \\ Funding \\ No fund. \\ Availability of data and materials \\ Not applicable.
}

The author T. Abdeljawad would like to thank Prince Sultan University for the moral support through the research lab TAS.

\title{
Declarations
}

Competing interests

The authors declare that they have no competing interests.

\section{Authors' contributions}

The main idea of this paper was proposed by TAA, TA, and DBP. TAA, MSA, MAA, and SSR prepared the manuscript initially and performed all the steps of the proofs in this research. All authors read and approved the final manuscript.

\section{Author details}

1 Department of Mathematics, Dr. Babasaheb Ambedkar Marathwada University, (M.S), Aurangabad, 431001, India. ${ }^{2}$ Department of Mathematics and Sciences, Prince Sultan University, Riyadh, Saudi Arabia. ${ }^{3}$ Department of Medical Research, China Medical University, Taichung, 40402, Taiwan. ${ }^{4}$ Department of Computer Science and Information Engineering, Asia University, Taichung, Taiwan. ${ }^{5}$ Department of Mathematics, Hodeidah University, Al-Hudaydah, Yemen. ${ }^{6}$ Department of Mathematics, Hajjah University, Hajjah, Yemen.

\section{Publisher's Note}

Springer Nature remains neutral with regard to jurisdictional claims in published maps and institutional affiliations.

Received: 26 September 2021 Accepted: 1 November 2021 Published online: 13 November 2021

\section{References}

1. Diethelm, K.: The Analysis of Fractional Differential Equations: An Application-Oriented Exposition Using Differential Operators of Caputo Type. Springer, Berlin (2010)

2. Kilbas, A.A., Srivastava, H.M., Trujillo, J.J.: Theory and Applications of Fractional Differential Equations. North-Holland Mathematics Studies. Elsevier, Amsterdam (2006)

3. Samko, S.G., Kilbas, A.A., Marichev, O.l., et al.: Fractional Integrals and Derivatives. Theory and Applications. Gordon \& Breach, Yverdon (1993)

4. Katugampola, U.N.: New approach to a generalized fractional integral. Appl. Math. Comput. 218(3), 860-865 (2011)

5. Caputo, M., Fabrizio, M.: Applications of new time and spatial fractional derivatives with exponential kernels. Prog. Fract. Differ. Appl. 2(1), 1-11 (2016)

6. Atangana, A., Baleanu, D.: New fractional derivatives with nonlocal and non-singular kernel: theory and application to heat transfer model. Therm. Sci. 20(2), 763-769 (2016)

7. Khan, H., Ibrahim, M., Abdel-Aty, A.H., et al.: A fractional order Covid-19 epidemic model with Mittag-Leffler kernel. Chaos Solitons Fractals 148, Article ID 111030 (2021). https://doi.org/10.1016/j.chaos.2021.111030

8. Khan, H., Abdeljawad, T., Gomez-Aguilar, J.F., et al.: Fractional order Volterra integro-differential equation with Mittag-Leffler kernel. Fractals 29(6), Article ID 2150154 (2021)

9. Tajadodi, H., Khan, Z.A., Gómez-Aguilar, J.F., Khan, A., Khan, H.: Exact solutions of conformable fractional differential equations. Results Phys. 22, Article ID 103916 (2021)

10. Khan, A., Alshehri, H.M., Abdeljawad, T., et al.: Stability analysis of fractional nabla difference Covid-19 model. Results Phys. 22, Article ID 103888 (2021). https://doi.org/10.1016/j.rinp.2021.103888

11. Aljaaidi, T.A., Pachpatte, D.B.: The Minkowski's inequalities via Riemann-Liouville fractional integral operators. Rend. Circ. Mat. Palermo (2) Suppl. 70, 893-906 (2021). https://doi.org/10.1007/s12215-020-00539-w

12. Beckenbach, E.F.: Generalized convex functions. Bull. Am. Math. Soc. 43, 363-371 (1937)

13. Dragomir, S.S.: On Hadamard's inequality for convex functions on the coordinates in a rectangle from the plane. Taiwan. J. Math. 4, 775-788 (2001)

14. Dragomir, S.S., Agarwal, R.P.: Two inequalities for differentiable mappings and applications to special means of real numbers and to trapezoidal formula. Appl. Math. Lett. 11(5), 91-95 (1998)

15. El Farissi, A., Latreuch, Z., Belaidi, B.: Hadamard-type inequalities for twice differentiable functions. RGMIA 12(1), Article ID 7 (2009) 
16. Florea, A., Niculescu, C.P.: A Hermite-Hadamard inequality for convex-concave symmetric functions. Bull. Math. Soc. Sci. Math. Roum. 50(98), 149-156 (2007)

17. Sarikaya, M.Z., Set, E., Yaldiz, H., Başak, N.: Hermite-Hadamard's inequalities for fractional integrals and related fractional inequalities. Math. Comput. Model. 57, 2403-2407 (2013)

18. Chen, F.X.: Extensions of the Hermite-Hadamard inequality for convex functions via fractional integrals. J. Math Inequal. 10(1), 75-81 (2016)

19. Liu, K., Wang, J., O'Regan, D.: On the Hermite-Hadamard type inequality for $\psi$-Riemann-Liouville fractional integrals via convex functions. J. Inequal. Appl. 2019, Article ID 27 (2019)

20. Nowicka, M., Witkowski, A.: Applications of the Hermite-Hadamard inequality. Math. Inequal. Appl. 19(4), 1319-1334 (2016)

21. Dahmani, Z:: On Minkowski and Hermite-Hadamard integral inequalities via fractional integration. Ann. Funct. Anal. 1(1), 51-58 (2010)

22. Dragomir, S.S., Fitzpatrik, S.: The Hadamard's inequality for s-convex functions in the second sense. Demonstr. Math. 32(4), 687-696 (1999)

23. Noor, M.A., Noor, K.I., Awan, M.U.: Some quantum estimates for Hermite-Hadamard inequalities. Appl. Math. Comput. 251, 675-679 (2015)

24. Agarwal, P., Jleli, M., Tomar, M.: Certain Hermite-Hadamard type inequalities via generalized k-fractional integrals. J. Inequal. Appl. 2017, Article ID 55 (2017). https://doi.org/10.1186/s13660-017-1318-y

25. Khan, M.A., Chu, Y.M., Kashuri, A., Liko, R., Ali, G.: Conformable fractional integrals versions of Hermite-Hadamard inequalities and their generalizations. J. Funct. Spaces 2018, Article ID 6928130 (2018). https://doi.org/10.1155/2018/6928130

26. Mohammed, P.O., Brevik, I.: A new version of the Hermite-Hadamard inequality for Riemann-Liouville fractional integrals. Symmetry 12, 610 (2020)

27. Awan, M.U., Akhtar, N., Iftikhar, S., et al.: New Hermite-Hadamard type inequalities for $n$-polynomial harmonically convex functions. J. Inequal. Appl. 2020, Article ID 125 (2020)

28. Chudziak, M., Ołdak, M.Z.: Hermite-Hadamard and Fejér inequalities for co-ordinated $(F, G)$-convex functions on a rectangle. Symmetry 12(1), Article ID 13 (2020). https://doi.org/10.3390/sym12010013

29. Khan, M.B., Mohammed, P.O., Noor, M.A., Abualnaja, K.M.: Fuzzy integral inequalities on coordinates of convex fuzzy interval-valued functions. Math. Biosci. Eng. 18(5), 6552-6580 (2021)

30. Delavar, M.R., Dragomir, S.S., De La Sen, M.: A note on characterization of $h$-convex functions via Hermite-Hadamard type inequality. Probl. Anal. Issues Anal. 8(26), 28-36 (2019)

31. Mohammed, P.: Hermite-Hadamard inequalities for Riemann-Liouville fractional integrals of a convex function with respect to a monotone function. Math. Methods Appl. Sci. 44(3), 2314-2324 (2019). https://doi.org/10.1002/mma.5784

32. Abdeljawad, T., Rashid, S., Khan, H., Chu, Y.-M.: On new fractional integral inequalities for $p$-convexity within interval-valued functions. Adv. Differ. Equ. 2020, 330 (2020)

33. Yan, P.Y., Li, Q., Chu, Y.M., et al.: On some fractional integral inequalities for generalized strongly modified $h$-convex function. AIMS Math. 5, 6620-6638 (2020)

34. Zhou, S.S., Rashid, S., Jarad, F., et al.: New estimates considering the generalized proportional Hadamard fractional integral operators. Adv. Differ. Equ. 2020, 1 (2020)

35. Jarad, F., Abdeljawad, T., Alzabut, J.: Generalized fractional derivatives generated by a class of local proportional derivatives. Eur. Phys. J. Spec. Top. 226, 3457-3471 (2017)

36. Jarad, F., Alqudah, M.A., Abdeljawad, T.: On more generalized form of proportional fractional operators. Open Math. 18, 167-176 (2020)

37. Aljaaidi, T.A., Pachpatte, D.B., Shatanawi, W., et al.: Generalized proportional fractional integral functional bounds in Minkowski's inequalities. Adv. Differ. Equ. 2021, Article ID 419 (2021). https://doi.org/10.1186/s13662-021-03582-8

38. Aljaaidi, T.A., Pachpatte, D.B.: Reverse Hermite-Hadamard's inequalities using $\psi$-fractional integral. Eng. Appl. Sci. Lett. 3, 75-84 (2020)

39. Caputo, M., Fabrizio, M.: A new definition of fractional derivative without singular kernel. Prog. Fract. Differ. Appl. 1(2), 73-85 (2015)

\section{Submit your manuscript to a SpringerOpen ${ }^{\mathcal{O}}$ journal and benefit from:}

- Convenient online submission

- Rigorous peer review

- Open access: articles freely available online

- High visibility within the field

- Retaining the copyright to your article

Submit your next manuscript at $>$ springeropen.com 